\section{Thyroglossal duct surgery. What is the acceptable recurrence rate?}

\author{
Asma A. Alahmadi, MD, SBORL, \\ Osama A. Bawazir, MBBS, FRCSC, \\ Mohannad K. Rajab, MD, SBORL, \\ Ibtihal A. Althobaiti, MD, \\ Abdullah O. Bawazir, $M B, B S$, \\ Firas R. Abi Sheffah, MD, ABORL, \\ Anas H. Al-Tammas, MD, ABORL, \\ Osama A. Marglani, MD, FRCSC, \\ John C. Heaphy, MD, ABOto,
}

Ameen Z. Alherabi, FACS, FRCSC.

\section{ABSTRACT}

Objectives: To present experiences of different specialties in the treatment of thyroglossal duct cysts (TGDCs) and subsequent complications in multiple centers.

Methods: A retrospective cross-sectional study of all cases of TGDC for a period of 11 years from 2008-2019 by different departments from 3 different centers in Jeddah, Kingdom of Saudi Arabia (King Faisal Specialist Hospital \& Research Centre, Bakhsh Hospital and International Medical Center).

Results: Forty-nine patients were included. The type of surgery performed plays a significant role in recurrence $(p<0.001)$. The Sistrunk procedure had a lower recurrence rate $(0 \%)$ than simple excision (70\%) and has showed a significantly long recurrencefree interval $(p<0.001)$. Higher recurrence rates are associated with higher postoperative complications $(p=0.002)$. Patients who underwent pre-operative fine needle aspiration did not have any recurrence during the follow-up period.

Conclusion: The Sistrunk procedure is the gold standard technique with the highest recurrencefree interval rate. Fine needle aspiration could be recommended as a less invasive procedure to exclude malignancy.

Keywords: thyroglossal duct cyst, recurrence, sistrunk, surgical

Saudi Med J 2020; Vol. 41 (8): 878-882 doi: 10.15537/smj.2020.8.25169

T he thyroglossal duct cyst (TGDC) is one of the most common congenital midline neck masses that are located at the level of or below the hyoid bone in approximately $80 \%$ of cases, and the remaining $20 \%$ are located above the hyoid bone. ${ }^{1}$ Epidemiologically, TGDC has a bimodal distribution in children and young adults. ${ }^{2}$ The clinical presentation of TGDC includes swelling, pain or tenderness, dysphagia, and dysphonia. ${ }^{3}$ However, inflammation or infection in the form of an abscess or cellulitis is the most common complication seen in TGDC patients. Other complications include the presence of the tract posterior to the hyoid bone or ectopic thyroid follicles. ${ }^{4}$ However, adults have been reported to experience more symptoms than children. ${ }^{2}$

Surgical removal is the definitive treatment; however, recurrence is a known possible outcome. ${ }^{3}$ To reduce the recurrence risk based on embryological principles, Schlange had suggested removal of the middle third of the hyoid bone with the main cyst; then, Sistrunk improved this technique further by removing the mid-portion of the hyoid bone and tissue between the hyoid bone and foramen cecum. ${ }^{5}$ However, the recurrence rate is still $6.6 \%$ in the pediatric population, irrespective of the type of surgery performed whether it is Sistrunk's operation or modified Sistrunk's procedure. ${ }^{6}$ Over time, it was found that the central neck dissection or anterior wide local excision to remove the entire thyroglossal tract remnant reduced the risk of initial failure and was considered a favorable option for the management of recurrent cases, especially in cases with a history of infected cyst or incision to avoid the risk of further recurrences. ${ }^{7}$

Few studies have emphasized the factors that are associated with TGDC recurrence that could increase the economic burden of the hospital and negatively influence the mental health of the patients and their family. The purpose of this study was to determine the factors related to TGDC recurrence and present the experiences of different specialties in the treatment of TGDCs and its complications in multiple centers.

Methods. A retrospective review of paper and electronic records were performed for all cases of TGDC for a period of 11 years from 2008 to 2019 by different departments (otolaryngology - head and neck surgery, pediatric surgery, and general surgery) from 3 different centers in Jeddah, Kingdom of Saudi Arabia (King Faisal Specialist Hospital \& Research Centre, Bakhsh Hospital, and International Medical Center).

We included all patients with the diagnosis of TGDC without restriction to age, nationality, or presenting symptoms. We excluded patients with missing documentation and also those who were lost to follow up of $<12$ months. Data extracted from patients' records included basic demographic characteristics, clinical presentation, medical history, postoperative variables, 
possible outcome parameters, and complications. All patients were followed up for 24 months after the surgery.

The literature review was carried out using PubMed, Medline, Embase, and Cochrane Library. Key terms related to thyroglossal duct cyst, recurrence, and Sistrunk were used to search for related published studies.

An institutional review board has approved this retrospective study and attests to its scientific validity. Informed written consent was provided by all participants in this study.

Statistical analysis. All statistical analyses were performed by the Statistical Package for Social Sciences for windows, version 25.0 (IBM Corp, Armonk, NY, USA). Descriptive statistics were formed from the data collected and expressed as mean, standard deviation (SD), and frequencies. Statistical comparisons between categorical variables were performed using Fisher's exact test or Chi-square test as appropriate. Kaplan-Meier (KM) curves were used to estimate the recurrence-free rate after surgery for TGDC. Moreover, a log rank test was performed to compare the recurrence-free rate among different procedures and specialists. A $p<0.05$ was considered significant.

Results. A total of 49 patients were included in the study; of these, $32.7 \%$ were male. The mean $\pm S D$ age of all patients was $11.8 \pm 11.4$, and most of them were Saudis $(83.7 \%)$. Only 5 patients $(10.2 \%)$ presented with compression symptoms, and 14 patients $(28.6 \%)$ had other comorbidities. Noteworthy, only 7 patients $(14.3 \%)$ had a previous surgery, which was in a different hospital than the ones included in our study and had no records, for the thyroglossal cyst, and most of them were male (71.4\%). Meanwhile, a total of 2 patients with papillary thyroid carcinoma (PTC) were reported, a 21-year-old male and a 22-year-old female. There was no significant difference in the examined characteristics between men and women (Table 1).

Association of recurrence with preoperative and operative variables. All patients with recorded recurrence $(\mathrm{n}=7)$ have been diagnosed with concomitant TGDC, and the cysts were situated either in the infrahyoid (71.4\%) or the suprahyoid (28.6\%). Regarding investigation modality, all patients

Disclosure. Authors have no conflict of interests, and the work was not supported or funded by any drug company.
Table 1 - Basic sociodemographic characteristics of the included patients.

\begin{tabular}{lccc}
\hline Variables & \multicolumn{2}{c}{ Gender } & $P$-value \\
& Female & Male & \\
\hline Age (years) & $4(25.0)$ & $6(18.2)$ & \\
$0-4$ & $3(18.8)$ & $6(18.2)$ & \\
$5-8$ & $3(18.8)$ & $6(18.2)$ & 0.979 \\
$9-12$ & $1(6.3)$ & $3(9.1)$ & \\
$13-16$ & $5(31.3)$ & $12(36.4)$ & \\
$>17$ & & & \\
Nationality & $1(6.3)$ & $7(21.2)$ & 0.184 \\
Non-Saudi & $15(93.8)$ & $26(78.8)$ & \\
Saudi & & & \\
Compression symptoms & $15(93.8)$ & $29(87.9)$ & 0.524 \\
No & $1(6.3)$ & $4(12.1)$ & \\
Yes & & & \\
Comorbidities & $14(87.5)$ & $21(63.6)$ & \\
No & $2(12.5)$ & $12(36.4)$ & \\
Yes & $14(87.5)$ & $28(84.8)$ & 0.804 \\
Previous surgery for the thyroglossl cyst & $5(15.2)$ & \\
No & $2(12.5)$ & \\
Yes & Values are presented as numbers and percentages (\%). \\
\hline \multicolumn{4}{l}{} \\
\hline
\end{tabular}

underwent ultrasound preoperatively. Moreover, no recurrence was detected in patients with a history of fine needle aspiration (FNA) or in those who had their surgery performed by a general surgeon. There was no significant effect for any of the pre-operative variables on the recurrence rates (Table 2).

For the operative factors, surgery duration $($ mean \pm SD) was slightly longer in the recurrence group $(90 \pm 43.8 \mathrm{~min})$ than that in the no-recurrence group $(85 \pm 59.5 \mathrm{~min})$. In addition, a total of 5 surgeons with an average minimum experience of $>15$ years performed the surgeries. Additionally, the patients who underwent a resection of the middle (central) part of hyoid bone showed no recurrence. The general surgery group had no recurrence rate in comparison to the pediatric group which was $13.3 \%$ while the otolaryngology - head and neck surgery group had the highest rate of $23.1 \%$. Nevertheless, there was no statistical significance found when comparing the data to those of the no-recurrence group (Table 2).

Association of recurrence with postoperative variables. All recurrent cases underwent a simple excision. A significant increase in risk of recurrence $(p<0.001)$ was found between simple excision and other procedures. Moreover, the recorded postoperative complications were significantly $(p=0.002)$ higher in the recurrence group $(71.4 \%)$ than in the nonrecurrence group (11.9\%). However, none of the other postoperative variables have showed any significant difference between the recurrence and no-recurrence groups (Table 3). 
Recurrence-free rates (procedure and specialty). For both combined Sistrunk's and total thyroidectomy and Sistrunk's alone, the KM curve showed a similar recurrence-free rate $(100 \%)$ during the follow-up period. In contrast, the simple excision procedure showed a significantly higher rate of recurrence $(p<0.001)$ according to the log-rank test.

In terms of the specialty performing the surgery, general surgery showed superiority, with no recurrent cases recorded in their sample. However, no statistical significance was found for this difference $(p=0.202)$ according to the log-rank test.

Discussion. This retrospective multicenter study describes our experience in TGDC management. Our study included 49 patients who underwent surgery for recurrent TGDC with histologically confirmed TGDC diagnosis in $86 \%(\mathrm{n}=42)$ of the patients or other possible differential diagnoses, such as dermoid cyst in $8 \%(n=3)$, multinodular goiter in $2 \%(n=1)$, PTC in
$2 \%(\mathrm{n}=1)$, or lymph node (LN) with metastasis in $2 \%$ $(\mathrm{n}=1)$ of cases.

Thyroglossal cyst recurrence is common and depends mainly on the type of surgery and the incomplete removal of TGDC. In a retrospective study with a large sample size of 207 patients, the overall recurrence rate was $9.7 \%$, and a significant difference was found among surgical types; the Sistrunk operation had a recurrence rate of $5.3 \%$, which was lower compared to that of plain excision at $55.6 \%{ }^{8}$ In another retrospective review on a large number of patients $(n=352)$, the overall recurrence rate was $4.5 \% .{ }^{9}$ By using a 3-dimensional reconstruction, it was shown that TGDC penetrated the hyoid bone as a result of the forward growth of the hyoid bone. This could possibly result in recurrence. ${ }^{10}$ Dissection of all tracts is recommended to decrease the risk of recurrence; however, dissection of the foramen cecum was not found to be as important as the partial dissection of the hyoid bone. ${ }^{9}$

Furthermore, central neck dissection is considered

Table 2 - Preoperative and operative variables stratified by incidence of recurrence.

\begin{tabular}{|c|c|c|c|c|c|}
\hline \multirow[t]{2}{*}{ Variables } & \multicolumn{4}{|c|}{ Recurrence } & \multirow[t]{2}{*}{$P$-value* } \\
\hline & & No & Yes & Total & \\
\hline \multicolumn{6}{|l|}{ Consistency } \\
\hline Cystic & 31 & $(73.8)$ & $7(100)$ & $38(77.6)$ & \multirow{2}{*}{0.124} \\
\hline Firm & 11 & $(26.2)$ & 0 & $11(22.5)$ & \\
\hline \multicolumn{6}{|l|}{ Location } \\
\hline Lingual & 1 & $(2.4)$ & 0 & $1 \quad(2.0)$ & \multirow{3}{*}{0.893} \\
\hline Suprahyoid & 10 & (23.8) & $2(28.6)$ & $12(24.5)$ & \\
\hline Infrahyoid & 31( & (73.81) & $5(71.4)$ & $36(73.5)$ & \\
\hline \multicolumn{6}{|l|}{ Prophylactic antibiotics } \\
\hline No & 8 & (19.1) & $1(14.3)$ & $9(18.4)$ & \multirow{2}{*}{0.619} \\
\hline Yes & 34 & $(81.0)$ & $6(85.7)$ & $40(81.6)$ & \\
\hline \multicolumn{6}{|l|}{ Pre-operative diagnosis } \\
\hline TGDC & 38 & $(90.5)$ & $7 \quad(100)$ & $45(91.8)$ & \multirow{2}{*}{0.528} \\
\hline TGDC with PTC & 4 & $(9.5)$ & 0 & $4 \quad(8.2)$ & \\
\hline \multicolumn{6}{|l|}{ Fine needle aspiration } \\
\hline No & 37 & $(88.1)$ & $7 \quad(100)$ & $44(89.8)$ & \multirow{2}{*}{0.446} \\
\hline Yes & 5 & (11.9) & 0 & $5(10.2)$ & \\
\hline \multicolumn{6}{|l|}{ Prophylactic antibiotics } \\
\hline No & 8 & $(19.1)$ & 1 (14.3) & $9(18.4)$ & \multirow{2}{*}{0.763} \\
\hline Yes & 34 & $(81.0)$ & $6(85.7)$ & $40(81.6)$ & \\
\hline \multicolumn{6}{|l|}{ Specialty } \\
\hline Otolaryngology - head \& neck surgery & 10 & $(23.8)$ & $3(42.9)$ & $13(26.5)$ & \multirow{3}{*}{0.592} \\
\hline General surgery & 6 & $(14.3)$ & 0 & $6(12.2)$ & \\
\hline Pediatric surgery & 26 & $(62.0)$ & $4(57.1)$ & $30(61.2)$ & \\
\hline Surgical duration $($ mean $\pm S D)$ & & \pm 59.53 & $90 \pm 43.79$ & $86 \pm 57.18$ & $0.835^{\dagger}$ \\
\hline \multicolumn{6}{|c|}{ Resection of the middle (central) part of hyoid bone } \\
\hline No & 3 & $(7.1)$ & 0 & $3(6.1)$ & \multirow{2}{*}{0.623} \\
\hline yes & 39 & $(92.9)$ & $7(100)$ & $46(93.9)$ & \\
\hline \multicolumn{6}{|l|}{ Re-approximation of the hyoid bone } \\
\hline No & 13 & $(31.0)$ & $3(42.9)$ & $16(32.7)$ & \multirow{2}{*}{0.412} \\
\hline Yes & 29 & $(69.1)$ & $4(57.1)$ & $33(67.4)$ & \\
\hline
\end{tabular}

Values are presented as numbers and percentages (\%). TGDC: thyroglossal duct cyst,

PTC: papillary thyroid carcinoma, SD: standard deviation, *Fisher's exact test, 'independent samples t-test 
Table 3 - Postoperative variables stratified by the incidence of recurrence.

\begin{tabular}{|c|c|c|c|c|}
\hline \multirow[t]{2}{*}{ Variables } & \multicolumn{3}{|c|}{ Recurrence } & \multirow[t]{2}{*}{$P$-value* } \\
\hline & No & Yes & Total & \\
\hline \multicolumn{5}{|l|}{ Treatment } \\
\hline Sistrunk procedure & $35(83.3)$ & 0 & $35(71.4)$ & \multirow{3}{*}{$<0.001^{\ddagger}$} \\
\hline Simple excision & $3(7.1)$ & $7(100)$ & $10(20.4)$ & \\
\hline Sistrunk procedure + total thyroidectomy & $4 \quad(95)$ & 0 & $4(8.2)$ & \\
\hline \multicolumn{5}{|l|}{ Drain placement } \\
\hline No & $35(83.3)$ & $5(71.43)$ & $40(81.6)$ & \multirow{2}{*}{0.381} \\
\hline Yes & $7(16.7)$ & $2(28.57)$ & $9(18.4)$ & \\
\hline \multicolumn{5}{|l|}{ Histopathology } \\
\hline Confirmed diagnosis & $35(83.3)$ & $7 \quad(100)$ & $42(85.7)$ & \multirow{6}{*}{1} \\
\hline Dermoid cyst ${ }^{\circ}$ & $3(7.1)$ & 0 & $3(7.1)$ & \\
\hline Inflamed dermoid cyst & $1 \quad(2.4)$ & 0 & $1(2.0)$ & \\
\hline MNG, no malignancy, TGDC & $1 \quad(2.4)$ & 0 & $1(2.0)$ & \\
\hline PTC, absence of TGDC & $1 \quad(2.4)$ & 0 & $1(2.0)$ & \\
\hline Level 1 L.N with metastatic PTC or TGDC & $1 \quad(2.4)$ & 0 & $1(2.0)$ & \\
\hline \multicolumn{5}{|l|}{ Postoperative complication } \\
\hline Fistula managed with antibiotics & $2(4.76)$ & $2(28.57)$ & $4(8.2)$ & \multirow{5}{*}{$0.002^{\dagger}$} \\
\hline Hematoma requiring surgical drainage & $0(0)$ & $1(14.29)$ & $1(2.0)$ & \\
\hline Keloid scar & $0(0)$ & $1(14.29)$ & $1(2.0)$ & \\
\hline None & $37(88.1)$ & $2(28.57)$ & $39(79.6)$ & \\
\hline Postoperative infection managed with antibiotics & $3(7.14)$ & $1(14.29)$ & $4(8.2)$ & \\
\hline \multicolumn{5}{|l|}{ Postoperative antibiotic } \\
\hline None & $2(4.76)$ & 0 & $2(4.2)$ & \multirow{6}{*}{0.827} \\
\hline Augmentin & $24(57.14)$ & $4(57.2)$ & $28(57.1)$ & \\
\hline Cefalexin & $7(16.67)$ & $2(28.6)$ & $9(18.4)$ & \\
\hline Cefazolin & $3(7.14)$ & 0 & $3(6.1)$ & \\
\hline Cefuroxime & $4(9.52)$ & 0 & $4(8.2)$ & \\
\hline Clindamycin & $2(4.76)$ & $1(14.3)$ & $3(6.1)$ & \\
\hline
\end{tabular}

Values are presented as number and percentages (\%). MNG: multinodular goiter, TGDC: thyroglossal duct cyst, PTC: papillary thyroid carcinoma, L.N: lymph node, ${ }^{*}$ Fisher's exact test, ${ }^{\dagger} p<0.05,{ }^{\dagger} p<0.001$

an alternative method in decreasing the recurrence rate. Isaacson et $\mathrm{al},{ }^{7}$ reported that central neck dissection could reduce the risk of initial failure in difficult cases, and even though it is a safe procedure, it should still be performed with caution to avoid the risk of injury to the carotid artery, vagus nerve, or larynx.

However, there are other risk factors that could increase the risk of recurrence. The rupture of the cyst could increase the risk of recurrence; however, in another study, the rupture of the cyst in 53 out of 159 cases was noted to have no effect on recurrences or postoperative complications, despite the extension of the cyst posterior to the hyoid. ${ }^{3,11}$ Furthermore, postoperative infection may increase the risk of recurrence. ${ }^{8}$ Other possible factors could be attributed to the recurrence, such as expertise of the surgeon and years of training, the persistence of infra or suprahyoid tract remnants, and a misdiagnosis of a TGDC. ${ }^{12}$

Furthermore, our result revealed that only 2\% were carcinomas or lymph nodes with metastasis. This finding is consistent with that of literature reporting that carcinoma could be present in approximately $1 \%$ of cases, but these cases had excellent prognosis if provided with adequate treatment. ${ }^{13}$ Moreover, in a retrospective analysis, PTC in the epithelium of the cyst was reported in $1.4 \%$ of cases. ${ }^{9}$ However, FNA is still favorable to confirm the diagnosis and distinguish malignant features; it was carried out in $10.2 \%$ of our cases. ${ }^{14}$ Contrary to malignant transformation, the incidence of ectopic thyroid in TGDC is unknown. Thus, preoperative imaging, such as sonography, computed tomography, and FNA biopsy are recommended as supplementary techniques to confirm the diagnosis. ${ }^{15}$ In our study, we did not identify any patient with ectopic thyroid tissue in the postoperative histopathological examination which other studies demonstrated as commensurate finding. ${ }^{9-11}$

Complications of the Sistrunk procedure are known to be minor ones and wound-related. ${ }^{15}$ In our study, we report no complications in 30/40 of patients who underwent Sistrunk operation, and in 39 out of the 49 patients in the total cohort. Moreover, other complications were related to infection and no statistically significant difference was found among the different types of surgery. In addition, the study has shown that surgeries conducted by general surgeons had a better prognosis as no recurrences were detected in cases that underwent general surgeries. These results 
are consistent with another retrospective study of 102 patients who were followed up for 14 years. ${ }^{15}$ The study showed that there was no recurrence detected in the general surgery group as compared to the 3\% recurrence rate found in the pediatric surgery group during the follow-up period. ${ }^{15}$ It is noteworthy that the pediatric surgery group $(n=67)$ was larger than the general surgery group $(n=35)$. Similarly, in our study, we only had 6 patients in the general surgery group, as compared to the 13 patients in the otolaryngology - head \& neck surgery group and 30 patients in the pediatric surgery group.

Study limitations. A small sample size with a short follow-up duration can make it hard to draw an efficient conclusion. Secondly, we included all age groups with no comparison regarding specific groups. Lastly, patients coming from 3 different centers and 3 different departments may give rise to variabilities in surgical techniques. Therefore, further multicenter studies with a larger sample size with more evaluation of the Sistrunk surgical procedure among all surgical specialties with focus on recurrent TGDC cases are strongly suggested.

In conclusion, there is a significant difference in the recurrence rates and postoperative complications among different types of surgeries. No significant superiority has been detected when comparing the different specialties in terms of recurrence rates. The Sistrunk procedure is the gold standard technique, showing a higher recurrence-free rate as compared to a simple excision. Fine needle aspiration is highly recommended as a non-invasive modality to exclude malignancy and improve outcomes.

Acknowledgment. The authors gratefully acknowledge Editage (www.editage.com) for English language editing.

Received 9th April 2020. Accepted 12th June 2020.

From the Department of Otolaryngology-Head \& Neck Surgery (Alahmadi, Rajab, Althobaiti, Abi Sheffah, Al-Tammas, Marglani, Heaphy, Alherabi); from the Department of Surgery (O. Bawazir), Pediatric Surgery Section, King Faisal Specialist Hospital \& Research Centre, from the College of Medicine (A. Bawazir), King Saud bin Abdulaziz University for Health Science, Jeddah, from the College of Medicine (O. Bawazir), Umm Al-Qura University; and from the Otolaryngology-Head \& Neck Surgery Department (Marglani, Alherabi), College of Medicine, Umm Al-Qura University, Makkah, Kingdom of Saudi Arabia.
Address correspondence and reprints request to: Dr. Ameen Z. Alherabi, Department of Otolaryngology-Head \& Neck Surgery, Umm Al-Qura University, Makkah,Kingdom of Saudi Arabia.Email:azherabi@uqu.edu.sa

ORCID ID: https://orcid.org/0000-0002-1001-3492

\section{References}

1. From the archives of the archives. Arch Ophthalmol 1989; 107: 786.

2. Brousseau VJ, Solares CA, Xu M, Krakovitz P, Koltai PJ. Thyroglossal duct cysts: presentation and management in children versus adults. Int J Pediatr Otorhinolaryngol 2003; 67: 1285-1290.

3. Mondin V, Ferlito A, Muzzi E, Silver CE, Fagan JJ, Devaney $\mathrm{KO}$, et al. Thyroglossal duct cyst: personal experience and literature review. Auris Nasus Larynx 2008; 35: 11-25.

4. Chandra RK, Maddalozzo J, Kovarik P. Histological characterization of the thyroglossal tract: implications for surgical management. Laryngoscope 2001; 111: 1002-1005.

5. Sistrunk WE. The surgical treatment of cysts of the thyroglossal tract. Ann Surg 1920; 71: 121-122.

6. Geller KA, Cohen D, Koempel JA. Thyroglossal duct cyst and sinuses: a 20-year Los Angeles experience and lessons learned. Int J Pediatr Otorhinolaryngol 2014; 78: 264-267.

7. Isaacson G. Central neck dissection for infected or recurrent thyroglossal duct cysts. Oper Tech Otolaryngol Head Neck Surg 2001; 12: 235-238.

8. Rohof D, Honings J, Theunisse HJ, Schutte HW, van den Hoogen FJ, van den Broek GB, et al. Recurrences after thyroglossal duct cyst surgery: results in 207 consecutive cases and review of the literature. Head Neck 2015; 37: 1699-1704.

9. de Tristan J, Zenk J, Künzel J, Psychogios G, Iro H. Thyroglossal duct cysts: 20 years' experience (1992-2011). Eur Arch Otorhinolaryngol 2015; 272: 2513-2519.

10. Horisawa M, Sasaki J, Niinomi N, Yamamoto T, Ito $T$. Thyroglossal duct remnant penetrating the hyoid bone--a case report. J Pediatr Surg 1998; 33: 725-726.

11. Taimisto I, Mäkitie A, Arola J, Klockars T. Thyroglossal duct cyst: patient demographics and surgical outcome of 159 primary operations. Clin Otolaryngol 2015; 40: 496-499.

12. Pelausa ME, Forte V. Sistrunk revisited: a 10-year review of revision thyroglossal duct surgery at Toronto's hospital for sick children. J Otolaryngol 1989; 18: 325-333.

13. Motamed M, McGlashan JA. Thyroglossal duct carcinoma. Curr Opin Otolaryngol Head Neck Surg 2004; 12: 106-109.

14. Yang YJ, Haghir S, Wanamaker JR, Powers CN. Diagnosis of papillary carcinoma in a thyroglossal duct cyst by fine-needle aspiration biopsy. Arch Pathol Lab Med 2000; 124: 139-142.

15. Al-Thani H, El-Menyar A, Sulaiti MA, El-Mabrok J, Hajaji $\mathrm{K}$, Elgohary $\mathrm{H}$, et al. Presentation, management, and outcome of thyroglossal duct cysts in adult and pediatric populations: a 14-year single center experience. Oman Med J 2016; 31 : 276-283. 